\title{
Y-Aminobutyric Acid Participates in the Adult-Phase Adventitious Rooting Recalcitrance
}

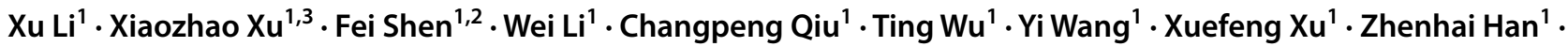 \\ Xinzhong Zhang ${ }^{1}$ (1)
}

Received: 24 February 2020 / Accepted: 14 October 2020 / Published online: 25 October 2020

(C) The Author(s) 2020

\begin{abstract}
In many tree species especially the rooting-recalcitrant woody perennials, the adventitious root (AR) in the juvenile phase can be easily induced by exogenous auxin, but AR formation becomes recalcitrant in the adult phase. Also, it is reported that the $\gamma$-aminobutyric acid (GABA) inhibits primary root growth in Arabidopsis and the AR formation in poplar (Populus ssp). So far, how GABA affects or is affected by the ontogenetic phase or auxin remains unclear. Here, we used an apple rootstock, Malus xiaojinensis, and tobacco (Nicotiana benthamiana) to investigate this question. We first analyzed the content of GABA, the activity of GABA synthetic enzyme GAD, and the expression of the coding gene MxGADs, respectively, in leafy cuttings of juvenile and adult phase. Next, the effect of exogenous GABA on AR formation was examined in in vitro shoots of $M$. xiaojinensis and tobacco. Interestingly, significant and consistent increases in GABA concentration, GAD activity, and expression of $M x G A D$ genes in response to exogenous indole butyric acid (IBA) were detected in adult-phase cuttings, but not in juvenile-phase cuttings. Exogenous GABA application inhibited the AR formation by delaying rooting time and reducing root number and the total root length in in vitro shoots of both M. xiaojinensis and tobacco. The expression of MxPIN members increased in response to IBA application, but these changes were restrained by the addition of GABA. These results indicate that both the loss of juvenility and IBA are required to trigger GABA accumulation. GABA may affect the AR formation as a co-actor by inhibiting polar auxin transport. Together, these findings facilitate the understanding of the regulatory network among GABA, juvenility, and auxin signaling on the AR formation.
\end{abstract}

Keywords Adventitious root $\cdot \gamma$-aminobutyric acid $\cdot$ Auxin $\cdot$ Juvenility $\cdot$ PIN

\section{Introduction}

$\gamma$-Aminobutyric acid (GABA), a non-protein amino acid, is synthesized from glutamate (Glu), which is catalyzed by the enzyme glutamate decarboxylase (GAD). Evolutionally,

Electronic supplementary material The online version of this article (https://doi.org/10.1007/s00344-020-10251-9) contains supplementary material, which is available to authorized users.

Xinzhong Zhang

zhangxinzhong999@126.com

1 College of Horticulture, China Agricultural University, Beijing, China

2 Beijing Agro-Biotechnology Research Center, Beijing Academy of Agriculture and Forestry Sciences, Beijing, China

3 College of Horticulture, Qingdao Agricultural University, Shandong, China
GABA is conserved from bacteria to animals, and was extensively studied in plants over half-a-century (Bouche et al. 2003; Bach et al. 2009; Podlesakova et al. 2019). GABA acts as an inhibitory neurotransmitter by regulating ion channels in mammalian central neural system (Behar et al. 1998). However, the function of GABA in plants has not been completely understood.

GABA is an important natural molecule produced in almost all living organisms, whereas its content is usually higher in plants than that in animals (Ramos-Ruiz et al. 2018; Al-Taher and Nemzer 2019). GABA accumulates in plants under abiotic stressful condition or undergoing senescence (Han et al. 2012; Zarei et al. 2015; Oh et al. 2019). For example, the GABA content in apple (Malus domestica) increased during cold storage or controlled atmosphere storage (low temperature and low oxygen) (Lum et al. 2016; Luo et al. 2018; Brikis et al. 2018). Under controlled atmosphere storage condition, the source of GABA is believed to 
be putrescine in addition to glutamate (Shelp et al. 2012). Moreover, copper-containing amine oxidase (MdCuAO1) oxidizes putrescine to produce GABA in the peroxisome of apple fruit under controlled atmosphere conditions (Zarei et al. 2015).

The role of GABA in plants can be quite diverse. GABA can not only regulate metabolic pathways like the Krebs cycle, but also act as a signaling molecule in plant growth and development (Bouche and Fromm 2004; Fait et al. 2008; Bown and Shelp 2016; Ramesh et al. 2017). Various abiotic or biotic stress stimuli induce an elevation of the GABA level in plant tissue (Bown and Shelp 1997; Kinnersley and Turano 2000; Che-Othman et al. 2020). Drought stressspecific responsive GABA accumulation induces stomatal opening and thereby prevents the water loss (Mekonnen et al. 2016). The exogenous application of GABA on rice (Oryza sativa) seedlings and black pepper (Piper nigrum) plants enhances the activity of enzymatic and non-enzymatic antioxidants, hence improving the growth and survival rate under heat, drought, or osmotic stress conditions (Nayyar et al. 2014; Vijayakumari and Puthur 2016). Exogenous GABA imparts salt tolerance by increasing proline and soluble sugar contents and upregulating antioxidants in maize (Zea mays) (Wang et al. 2017). GABA priming in rice germinating seeds significantly alleviates the effects of osmotic and salinity stress on seed germination and seedling growth, by inducing antioxidant activities (Sheteiwy et al. 2019). In addition, GABA application was shown to promote the growth of maize or muskmelon (Cucumis melo) under abiotic stress by reducing the production of reactive oxygen species (ROS), activating antioxidant activity, and improving photosynthesis (Salah et al. 2019; Jin et al. 2019). Besides, the effects of GABA in plant carbon and nitrogen metabolism, its responses to biotic and abiotic stress factors, in the improvement of shelf life and storage quality, and in maintaining $\mathrm{C} / \mathrm{N}$ balance and cytosolic $\mathrm{pH}$ have been discussed in previous reviews (Bouche and Fromm 2004; Ramos-Ruiz et al. 2019). Several studies revealed that GABA is also associated with the developmental processes in plant such as pollen tube growth, stem growth, and primary root growth (Palanivelu et al. 2003; Akama and Takaiwa 2007; Renault et al. 2011).

Adventitious root (AR) formation is crucial for plant growth and adaptation to various environmental conditions. The ability of AR formation is the most important requisite for vegetative propagated fruit and forestry species ( $\mathrm{Li}$ et al. 2017; Liu et al. 2020; Rasmussen et al. 2015). Exogenous and endogenous factors including phytohormones, environments, and plant age determine the AR formation (da Costa et al. 2013; Dawood et al. 2016; Druege et al. 2019). Auxin can be transported to AR competent cells by polar auxin transporters, such as PIN-FORMRD (PIN) and ATP-BINDING CASSETTE B19 (ABCB19) (Xu 2017; Sukumar et al.
2013; Liu et al. 2014). In several AR formation recalcitrant species such as Malus, the loss of juvenility causes a considerable reduction in AR formation ability, which is regulated by miR 156 and SQUAMOSA PROMOTER BINDING PROTEIN-LIKE (SPL) module. (Rasmussen et al. 2015; Xu et al. 2016, 2017). In M. xiaojinensis, which can be used as rootstock of apple trees (M. domestica), MxSPL26 is the dominant target of miR156 associated with AR formation (Xu et al. 2017). Rejuvenated in vitro shoots via repeated subculture of adult stock materials can restore the miR156 level and re-acquire the ability of AR formation (Xiao et al. 2014). The overexpression of miR 156 leads to increased AR production, and 35:MIM156 transformant produces fewer roots in Arabidopsis (Xu et al. 2016).

GABA is reported to affect the AR formation and elongation as well. In a GABA catabolism defective mutant of Arabidopsis, GABA accumulation causes defects of cell elongation and subsequent inhibition on primary root growth (Renault et al. 2011). Similarly, exogenous GABA inhibits primary root growth in Arabidopsis (Renault et al. 2011). Recently, it was reported that the inhibition of AR growth by blocking $\alpha$-ketoglutarate dehydrogenase ( $\alpha$-KGDH) was accompanied by changes in GABA shunt (Yue et al. 2018). In poplar (Populus alba $\times$ P. glandulosa), endogenous GABA accumulation not only inhibited the AR growth, but also delayed AR primordia formation and the AR appearance within one day. This delay was associated with changes in ethylene, auxin, and abscisic acid levels as well as their signaling (Xie et al. 2020).

Till date, it is not known whether GABA production is associated with juvenility and/or exogenous auxin in affecting $\mathrm{AR}$ formation. To address this question, the expression of $M x G A D s$ was analyzed in leafy cuttings of juvenile- and adult-phase $M$. xiaojinensis, and the effect of exogenous GABA on AR formations was also examined. The results may advance our understanding the role of GABA in plant AR formation.

\section{Material and Methods}

\section{Plant Materials and Treatments}

In vitro shoots of M. xiaojinensis and tobacco (Nicotiana benthamiana) (from seeds) were used for in-room treatments. M. xiaojinensis represents adult-phase rootingrecalcitrant woody perennial (Xu et al. 2017). To examine whether the effect of GABA on AR formation is speciesspecific or common among plants, tobacco was also used in this study as an easy-to-root annual herbaceous plant. The explants of $M$. xiaojinensis were shoot tips from oneyear-old seedlings, after $\sim 15$ passages of stem subculture for proliferation (Xiao et al. 2014). The in vitro shoots were 
cultured on Murashige \& Skoog (MS) medium containing $0.5 \mathrm{mg} / \mathrm{L}$ indole butyric acid (IBA) (V900325, SigmaAldrich, St. Louis, MO, USA), $0.3 \mathrm{mg} / \mathrm{L}$ 6-benzylamino purine (6-BA) (CAS 1214-39-7, Sigma-Aldrich, USA), 1.0\% agar, and $30 \mathrm{~g} / \mathrm{L}$ sucrose. The photoperiod was set with $16 \mathrm{~h}$ light $\left(22^{\circ} \mathrm{C}\right)$ and $8 \mathrm{~h}$ dark $\left(22^{\circ} \mathrm{C}\right)$. The rooting medium was $1 / 2 \mathrm{MS}$ plus $0.5 \mathrm{mg} / \mathrm{L}$ IBA. For GABA treatments, 0.00 , $0.25,1.0,4.0$, and 16.0 mM GABA (CAS 56-12-2, Sigma, USA) were added into the rooting medium, respectively. Experiments were performed based on a complete randomized design of three biological replicates.

Semi-lignified leafy cuttings $(8-10 \mathrm{~cm}$ length) from $M$. xiaojinensis basal suckers (juvenile phase, $\mathrm{Mx}-\mathrm{J}$ ) were used in greenhouse trial (Xiao et al. 2014; Xu et al. 2017). After excision, the base of leafy cuttings was immediately soaked in $3000 \mathrm{mg} / \mathrm{L} \mathrm{IBA}$ solution for $1.0 \mathrm{~min}$, with the IBA-free solution as a control (Xiao et al. 2014; Xu et al. 2017). GABA was added into the IBA solution with the concentrations of $0.0,5.0,50.0$, and $500.0 \mathrm{mM}$. Then, the cuttings were plugged into 50 cell trays and incubated in a misty solar greenhouse (Xiao et al. 2014; Xu et al. 2017). The ARs were measured five weeks after cutting. A complete randomized experiment design was used with three biological replicates (50 cuttings per replicate).

RNA-seq was previously performed using leafy cuttings from the adult-phase $M$. xiaojinensis (Mx-A) and $\mathrm{Mx}-\mathrm{J}$. The cutting was treated with $3000 \mathrm{mg} / \mathrm{L}$ IBA, with water as control. Bark samples were collected from $1.0 \mathrm{~cm}$ basal part of the cuttings at $0,6,12$, and $24 \mathrm{~h}$ after IBA treatment, respectively. The experiment was designed with three biological and three technical replicates. The complete dataset will be published separately and the data can be consulted at PRJNA392909.

\section{Measurement of Rooting}

The AR percentage was scored every two days. The final rooting percentage, length of roots, and number of roots per plant were examined at the fifth week after cutting or microcutting. At the same time, photographs were taken. The photos were then processed with Image $\mathbf{J}$ software.

\section{Gene Expression Analysis}

The expression profiles of $M x G A D s$ during the AR formation were analyzed based on the time-series of RNA-seq data. The relative expression of MxGADs and MxPINs was analyzed using real-time qPCR. The gene-specific primers were designed using Primer software (version 5.0) (Table S1). Thirteen PIN gene family members, that showed detectable expression in apple stem or shoot apex, were selected for qPCR analysis (https://bioinformatics.cau.edu.cn/Apple $\mathrm{MDO} / g e n e \_f a m i l y / f a m i l y \_m e m b e r . p h p ? g e n e \_f a m i l y=T r a n s$
porter\&\&family_id=AEC) (Da et al. 2019). One microgram of total RNA was used in reverse transcription in a total volume of $20 \mu \mathrm{L}$. Real-time qPCR was performed in $20 \mu \mathrm{L}$ system, containing $10 \mu \mathrm{L} 2 \times \mathrm{SYBR}$ Green Master mix (Takara, Japan), $300 \mathrm{mM}$ each primer, and $2 \mu \mathrm{L}$ tenfold diluted cDNA template. The PCR reactions were performed in ABI QuantStudio $^{\text {TM }} 6$ PCR Detection System (Thermofisher Applied Biosystems, USA) using the following program: $95^{\circ} \mathrm{C}$ for $30 \mathrm{~s}$ and 40 cycles of $95^{\circ} \mathrm{C}$ for $5 \mathrm{~s}$, annealing at $60^{\circ} \mathrm{C}$ for $30 \mathrm{~s}$. Triplicates of each reaction were conducted, and the $M d E F 1 \alpha$ gene was used as an internal control for normalization. Data were presented in a format of mean \pm standard deviation after normalization.

\section{Determination of GAD Activity}

GAD enzymatic activity was evaluated as described in Lü, et al. (2010). One gram of plant tissue was used for GAD extraction. The extraction mixture was consisted of $10 \mathrm{~mL}$ of $80 \mathrm{mM}$ sodium phosphate ( $\mathrm{pH}$ 5.6), $100 \mathrm{mM}$ L-glutamate, and $0.2 \mathrm{mM}$ pyridoxal phosphate (PLP). The mixture was incubated at $40{ }^{\circ} \mathrm{C}$ for $60 \mathrm{~min}$, and then terminated by a water bath at $90{ }^{\circ} \mathrm{C}$ for $5 \mathrm{~min}$. After centrifugation (3000 rpm, $10 \mathrm{~min}$ ), the GABA in the supernatant was assayed. The GAD activity was defined as units of GAD activity per gram of plant tissue. One unit of GAD activity was defined as a release of $1 \mathrm{mM}$ of GABA produced from glutamate per $30 \mathrm{~min}$ at $40{ }^{\circ} \mathrm{C}$.

\section{Derivatization and Quantification of GABA}

$1 \mathrm{~mL}$ of standard GABA solution or sample was used for GABA derivatization. 1 mLof $0.5 \mathrm{M} \mathrm{NaHCO}_{3}(\mathrm{pH} 9.0)$ and $1 \mathrm{~mL}$ of 2,4-dinitrofluorodinitrobenzene (FDNB) (1\%) reagent were added to a $10-\mathrm{mL}$ volumetric flask. The mixture was vortex-mixed for $5 \mathrm{~s}$ and kept in dark at $60{ }^{\circ} \mathrm{C}$ for $1 \mathrm{~h}$. After cooling to room temperature $\left(20-25^{\circ} \mathrm{C}\right)$, phosphate buffer $(0.02 \mathrm{M}, \mathrm{pH} 7.0)$ was added to the scale line of the flask. After mixing, the solution was filtered through a $0.22 \mu \mathrm{m}$ membrane filter (Whatman, USA). The filtrate was analyzed soon by high-performance liquid chromatography (HPLC) to determine the GABA concentrations.

The HPLC analysis was performed according to the method described in Lü et al. (2010). The determination of a derivative from the samples was carried out by HPLC (ACQUITY Arc system, Waters, Milford, USA) with a $2489 \mathrm{UV} / \mathrm{V}$ is detector (Waters, Milford, USA) at $360 \mathrm{~nm}$. A reversed-phase column (Waters, $\mathrm{C} 18,250 \mathrm{~mm} \times 4.6 \mathrm{~mm}$ ) was used for HPLC. Different concentrations of GABA (CAS 56-12-2, Sigma, USA) with 2,4-dinitrofluorodinitrobenzene (FDNB) were used to generate standard curves for quantification. 
A

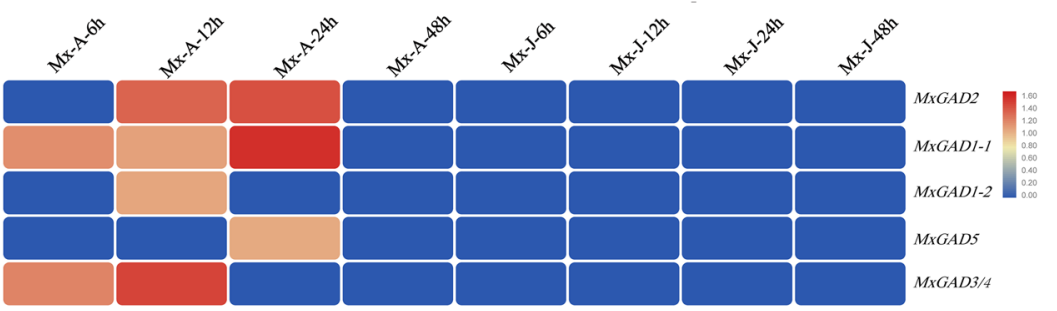

C

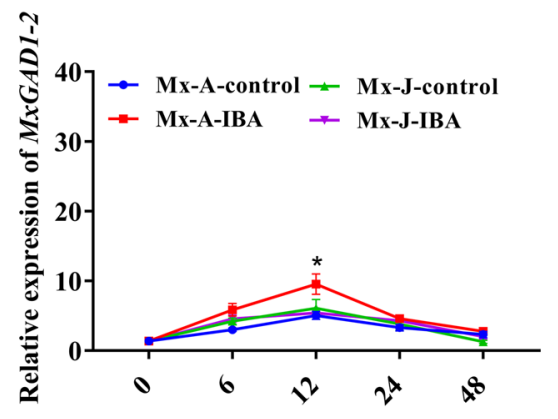

Duration post-treatment (hours)

$\mathbf{F}$

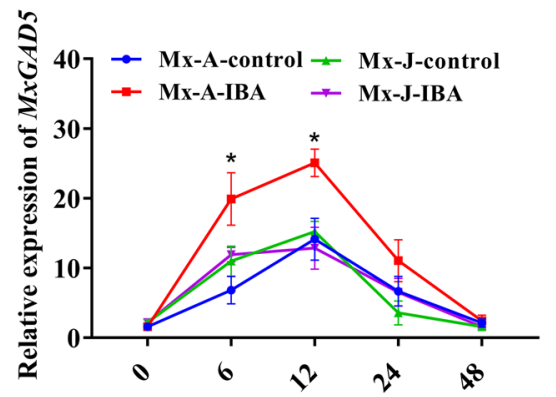

Duration post-treatment (hours)

I
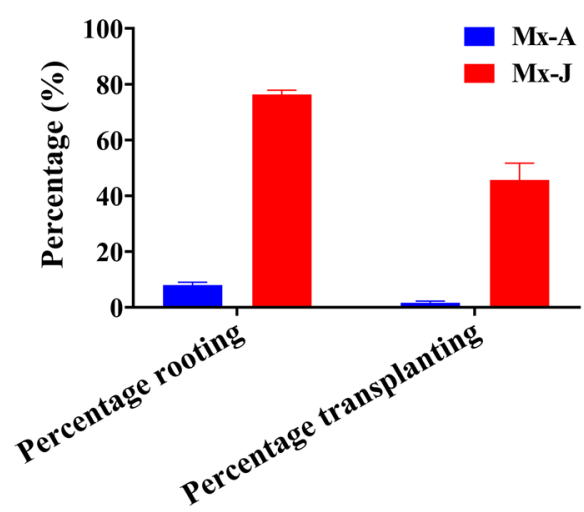

B

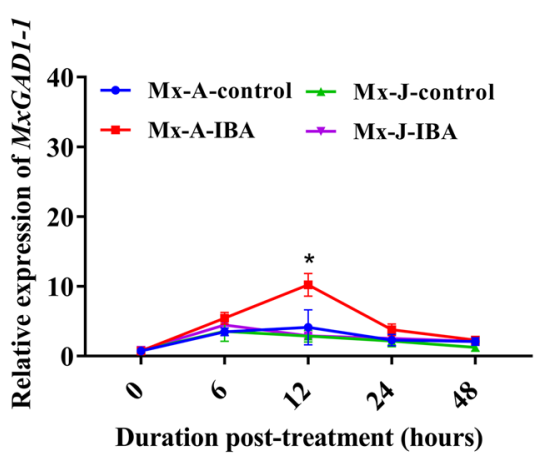

E

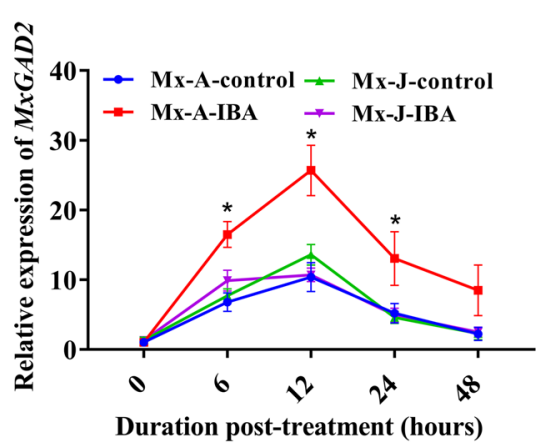

H

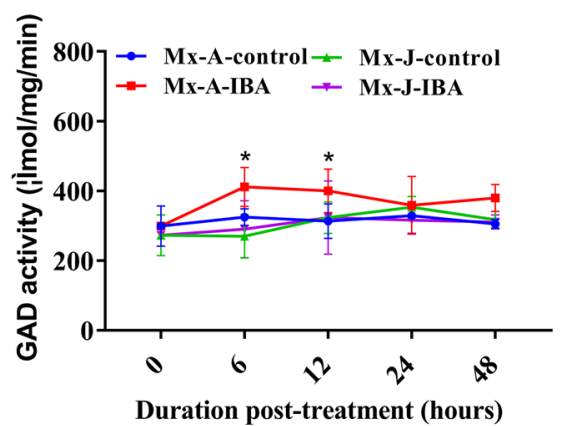

Duration post-treatment (hours)

G
Duration post-treatment (hours)

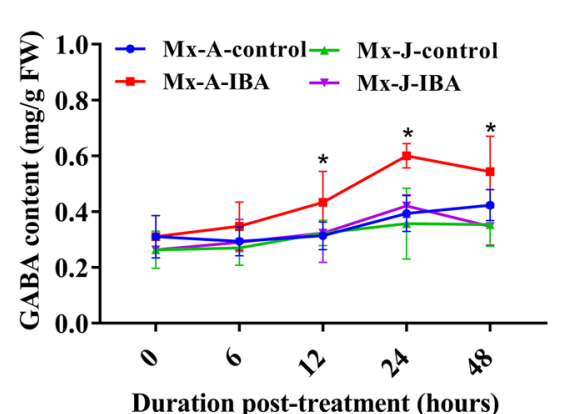

Duration post-treatment (hours)

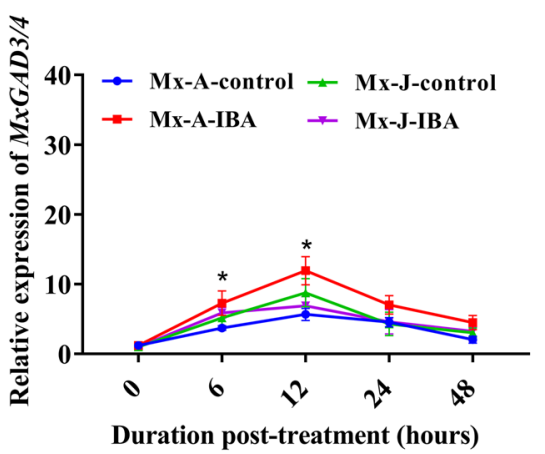

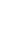


४Fig. $1 \gamma$-Aminobutyric acid (GABA) content, glutamate decarboxylase (GAD) activity, and $M x G A D s$ expression in leafy cuttings of the juvenile (Mx-J)- and adult (Mx-A)-phases Malus xiaojinensis. a Expression profiles of $M x G A D s$ by fragments per kilobase per million (FPKM) detected by a previous RNA-seq. $\mathbf{b}-\mathbf{f}$ Relative expression of the five $M x G A D s$ detected by qPCR during adventitious root (AR) formation. $\mathbf{g}$ Changes in GAD enzyme activity during AR formation. h Changes in GABA content during AR formation. $\mathbf{i}$ The percentage of rooting and transplanting at the fifth week after cutting for Mx-J and $\mathrm{Mx}-\mathrm{A}$

\section{Statistical Analysis}

Analysis of variance (ANOVA) was performed using the Statistical Product and Service Solutions (SPSS) software (IBMCo., Armonk, USA). All experimental data were tested by ANOVA test.

\section{Results}

\section{Analysis of MdGADs Expression Using Previous RNA-Seq Data}

From the RNA-seq data, five $M x G A D$ genes exhibited differential expression between $\mathrm{Mx}-\mathrm{A}$ and $\mathrm{Mx}-\mathrm{J}$ cuttings. These genes were designated as MxGAD1-1, MxGAD1-2, $M x G A D 2, M x G A D 3 / 4$, and $M x G A D 5$ according to the phylogenesis with Arabidopsis (Fig. S1). All the five MxGADs were significantly upregulated at 6-24 h after cutting in Mx-A cuttings in response to IBA (Fig. 1a). However, in $\mathrm{Mx}-\mathrm{J}$, no significant change of $M x G A D s$ expression was detected throughout the experiment (Fig. 1a).

The expression patterns of these $M x G A D s$ were then validated through qPCR (Fig. 1b-f). The relative expression of all the five $M x G A D$ s, especially $M x G A D 2$ and $M x G A D 5$, was considerably induced in Mx-A cuttings $6 \mathrm{~h}, 12 \mathrm{~h}$, or $24 \mathrm{~h}$ after IBA treatment (Fig. 1b-f). The GAD enzyme activity was apparently higher in Mx-A cuttings 6-12 h after IBA treatment (Fig. 1g). Consistently, the GABA contents were significantly higher in Mx-A cuttings 12-48 h after IBA treatment (Fig. 1h). Neither GAD activity nor GABA content varied in Mx-J, regardless of IBA addition (Fig. 1g, h). In the absence of IBA, no considerable variations were detected in GABA content, GAD enzyme activity, and $M x G A D s$ expression between $\mathrm{Mx}-\mathrm{J}$ and $\mathrm{Mx}-\mathrm{A}$.

At the fifth week after cutting, higher the rooting percentage $(76 \%)$ and transplanting percentage (more than three roots per plant) (45\%) were obtained in IBA-treated Mx-J cuttings. In comparison, the rooting percentage $(8 \%)$ and transplanting percentage (2\%) were very low for Mx-A cuttings (Fig. 1i). Together, GABA accumulated in Mx-A cuttings in response to IBA treatment.

\section{Effects of Exogenous GABA on Adventitious AR Formation}

To further test the effect of exogenous GABA on adventitious AR formation, we applied GABA to in vitro shoots of tobacco, in vitro shoots and greenhouse leafy cutting of M. xiaojinensis. At day 18 of GABA treatment, ARs were merely observed in vitro shoots of $M$. xiaojinensis in the control and $0.25 \mathrm{mM}$ GABA treatment (Fig. 2a). In comparison, the percentage of AR formation was significantly lower in $0.25 \mathrm{mM}$ GABA-treated group than that in control (Fig. 2a). No AR was found in in vitro shoots of 1.0, 4.0, and $16.0 \mathrm{mM}$ GABA treatments (Fig. 2a). At day 20 and 22, ARs emerged in GABA-treated in vitro shoots; however, the rooting percentage was still lower in GABA treatments with higher concentrations (Fig. 2a). At day 24 and 26, the percentage of rooted in vitro shoots in GABA treatments displayed similar levels as that in the control (Fig. 2a). At day 26 , however, the number of roots per plant and root length per plant was significantly lower at dose-dependent manner in GABA-treated in vitro shoots than that in the control (Fig. 2b, c).

Consistent results were observed in tobacco. After day 9-11, significantly less ARs were formed in tobacco in vitro shoots with higher concentrations of GABA than that in the control (Fig. 3a). While at day 13-17, these differences of rooting percentage disappeared regardless of GABA concentrations (Fig. 3a). At day 17, the number of roots per plant and root length per plant was considerably reduced in GABA treatments at dose-dependent manners (Fig. 3b, c).

In the greenhouse trial, the cuttings rooted very poorly when treated with absence of IBA. The percentages of rooting and transplanting did not vary between IBA + GABA treatments and IBA solely, or among different GABA concentrations (Fig. 4a). However, the number of roots per plant and root length per plant was significantly lower when treated with high concentrations of GABA than that with low GABA concentrations or IBA only (Fig. 4b, c).

Together, GABA inhibited AR formation by delaying rooting time and reducing AR quantity in both $M$. xiaojinensis and tobacco.

\section{Effect of Exogenous GABA on Polar Auxin Transport During AR Formation}

To test whether GABA affects the polar auxin transport, changes in the expression of MxPIN members were assessed during the AR formation in in vitro shoots of $M$. xiaojinensis. The phylogenetic relationship between the 13 PIN gene family members (MdPIN1-MdPIN13) in apple and those in Arabidopsis was analyzed based on the alignment of their amino acid sequences (Fig. S2). Of the MxPIN gene members, the relative expression of $M x P I N 1, M x P I N 3, M x P I N 4$, 
A

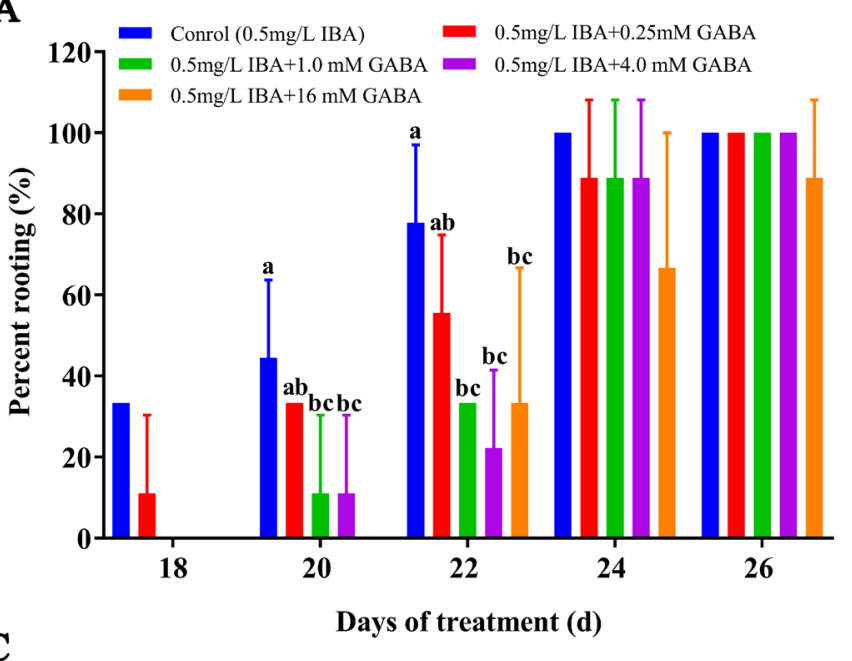

C

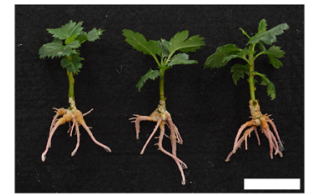

0 mM GABA

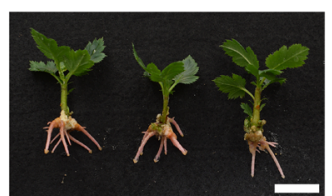

$0.25 \mathrm{mM} \mathrm{GABA}$

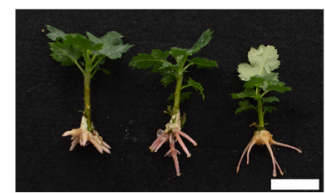

1.0 mM GABA

B

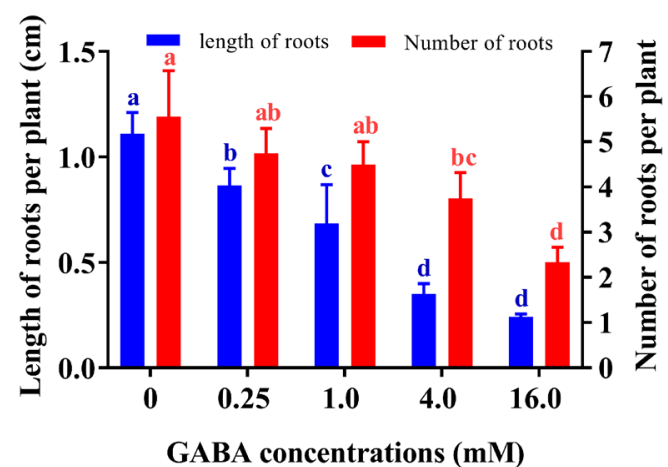

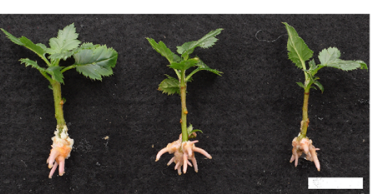

4.0 $\mathrm{mM} \mathrm{GABA}$

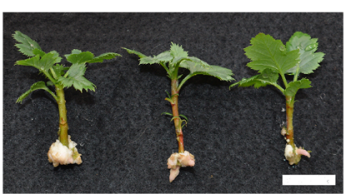

16.0 mM GABA

Fig. 2 Effects of exogenous $\gamma$-aminobutyric acid (GABA) on adventitious root formation in in vitro shoots of Malus xiaojinensis. a Dynamic changes in percent rooting. b Differences in root number and root length per plant at day 24. $\mathbf{c}$ Differences in adventitious rooting at day 24

MxPIN9, MxPIN10, and MxPIN12 was relatively higher in stem tissue of Mx-J cuttings (Fig. S3). When IBA was added to the culture medium without GABA, the expression of MxPIN1, MxPIN3, MxPIN4, and MxPIN9 was obviously unregulated at 24-96 h (Fig. 5a-d). No robust significant change was found in MxPIN10 or MxPIN12 expression (Fig. 5e, f). In contrast, the addition of GABA to the medium restrained the changes for all MxPIN members (Fig. 5a-f). These data clearly indicated that GABA inhibited the auxininduced upregulation of MxPINs.

\section{Discussion}

In several woody perennials, AR formation recalcitrance occurs when a plant enters adult phase of ontogenesis but ARs can be easily induced by exogenous auxin in juvenile phase or rejuvenated materials (Xiao et al. 2014; Porfirio et al. 2016; Xu et al. 2017). This adult-phase AR formation recalcitrance has been proven to be attributed to miR156SPL regulation pathway (Xiao et al. 2014; Xu et al. 2017). In this study, by using $M$. xiaojinensis leafy cuttings from the juvenile and adult phases, no difference in GABA content and biosynthesis was detected between ontogenetic phases under regular condition (Fig. 1b-h). Therefore, the adult phase, characterized by low miR156 and high SPL levels (Jia et al. 2017; Xu et al. 2017), might not be sufficient for
GABA accumulation. It's solid that exogenous IBA induced AR formation (Druege et al. 2019). In Mx-A, exogenous IBA did not promote AR formation (Xu et al. 2017), but IBA induced GABA accumulation. Similarly, previous studies reported that in root cultures of Datura stramonium, the addition of $\alpha$-naphthaleneacetic acid (NAA) led to 3- to-4 folds increase in GABA biosynthesis (Ford et al. 1996). The GABA content also changed in response to exogenous indole-3-acetic acid (IAA) treatment in roots of Arabidopsis (Anegawa et al. 2015). This adult-phase-specific IBAinduced GABA accumulation was hence suspected to be an important factor involved in the adult-phase-specific AR recalcitrance.

The expression of $M x G A D s$ and GAD enzyme activity in Mx-A increased since $6 \mathrm{~h}$ and subsequently GABA content increased since $12 \mathrm{~h}$ in response to exogenous IBA application. The discrepancy would be attributed to the larger variances between replicates and the relatively low concentration of GABA in the semi-lignified stem tissue (Mustroph et al. 2014; Oh et al. 2019).

Conversely, exogenous GABA treatments may alter auxin biosynthesis, transport, and signaling during AR formation. In the GABA-treated poplar in vitro stem segments, the IAA contents increased at day 1-3, whereas gene members of $Y U C, G H 3, A B P, A u x / I A A$, and $A R F$ were differentially expressed during AR formation (Xie et al. 2020). Similarly, at the early stage of AR formation, the concentrations of 
A

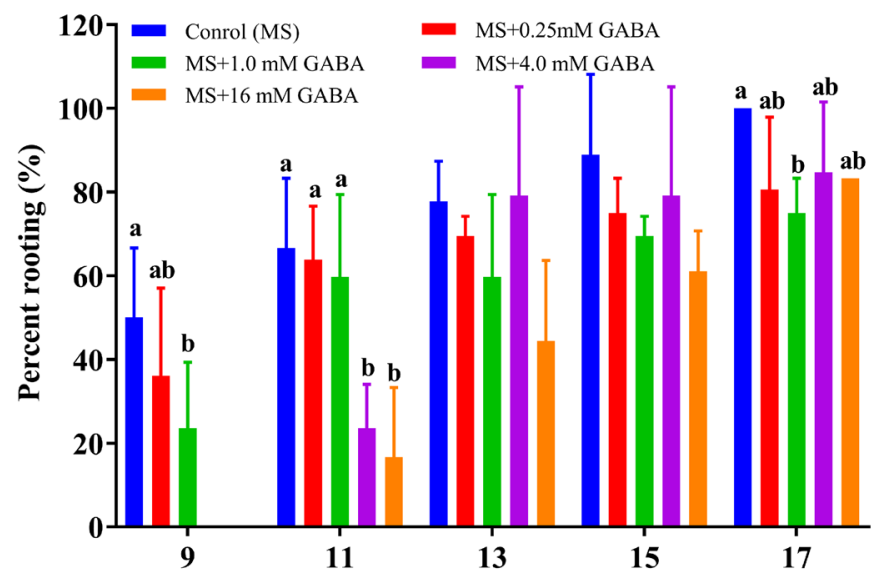

B

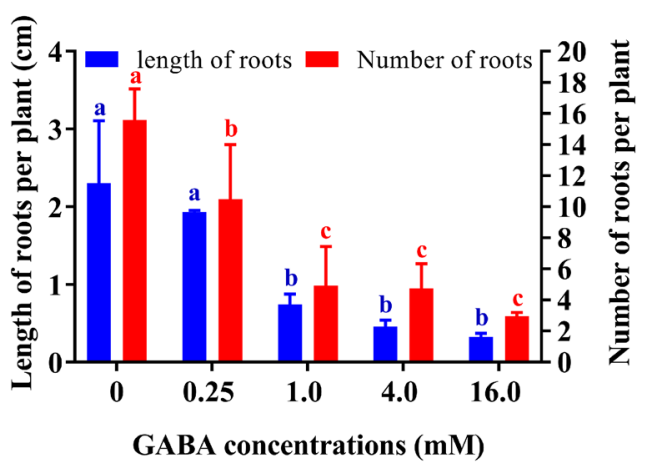

GABA concentrations $(\mathrm{mM})$

Days of treatment (d)
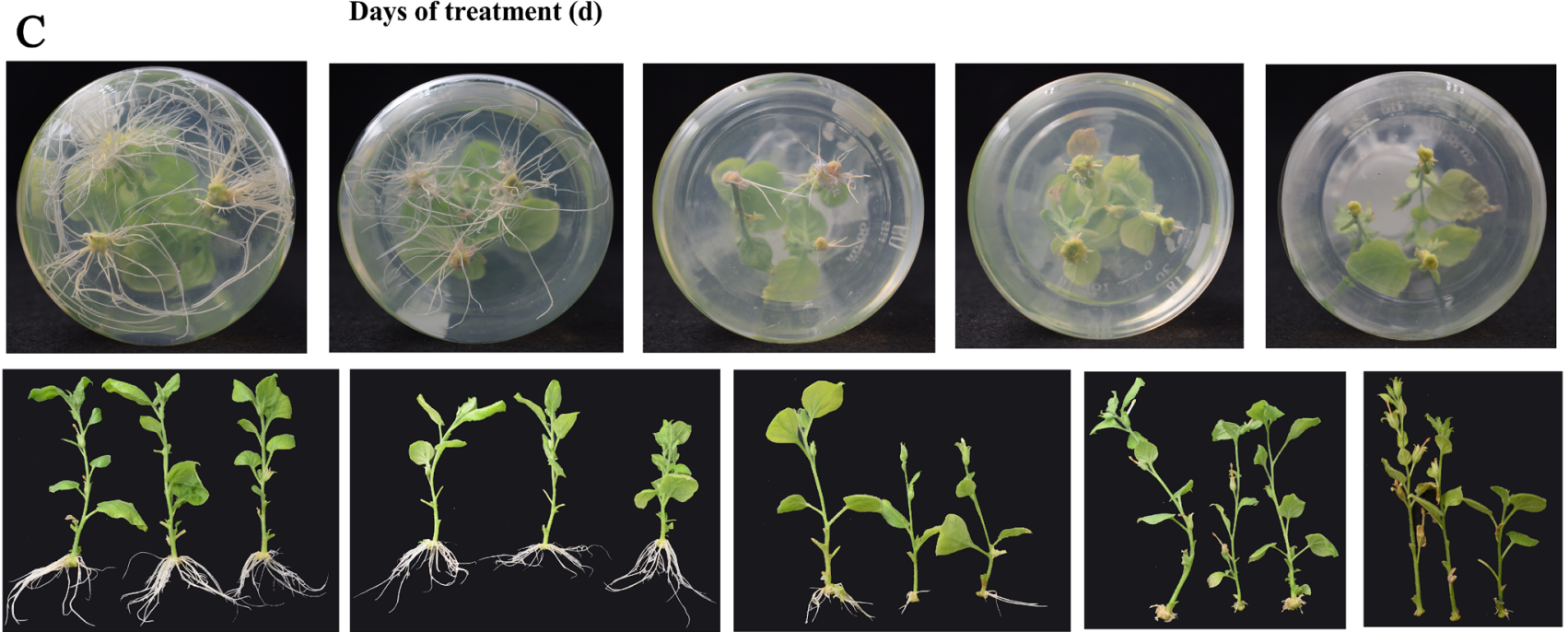

0 mM GABA

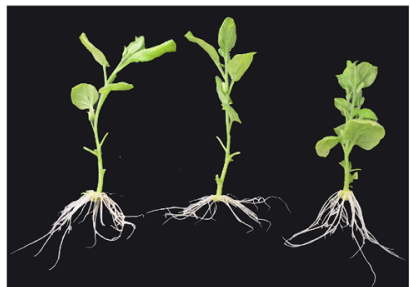

$0.25 \mathrm{mM}$ GABA

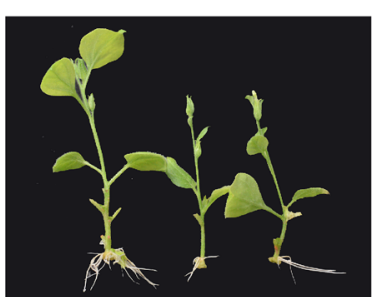

$1.0 \mathrm{mM}$ GABA

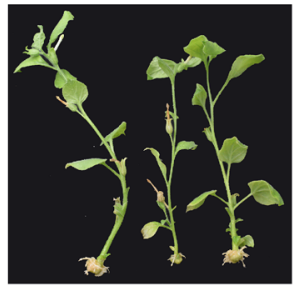

4.0 $\mathrm{mM} \mathrm{GABA}$

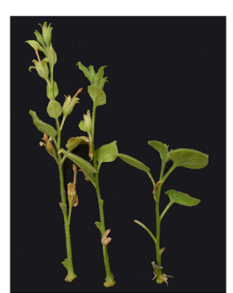

$16.0 \mathrm{mM}$ GABA
Fig. 3 Effects of exogenous $\gamma$-aminobutyric acid (GABA) on adventitious root formation in in vitro shoots of Nicotiana benthamiana. a Dynamic changes in percent rooting. b Differences in root number

total flavonoids and rutin increased after GABA treatment in poplar (Xie et al. 2020), and high levels of flavonoids and rutin may have affected polar auxin transport (Druege et al. 2019). In this study, we also found that the exogenous GABA application affected the expression of MxPINs and delayed AR formation.

PIN family members, especially the plasma membrane localized PIN proteins with a long hydrophilic central loop (PIN1, PIN2, PIN3, PIN4, and PIN7), were shown to catalyze auxin efflux at cellular level in Arabidopsis (Krecek et al. 2009; Ganguly et al. 2010). These auxin efflux carriers, like PIN1, are required for lateral root development in Arabidopsis (Benkova et al. 2003). In apple, MxPIN1, MxPIN9, and MxPIN10 showed close sequence similarity with Arabidopsis PIN1 (Fig. S2). Apple MdPIN1b protein was also confirmed to be localized on plasma membrane (Gan et al. and root length per plant at day 17. c Differences in adventitious rooting at day 17

2018). We observed in this study that the expression of MxPIN1, MxPIN3, MxPIN4, and MxPIN9 in in vitro shoots of Mx-J increased in response to IBA application, but the addition of exogenous GABA counteracted these increases (Fig. 5). We previously reported that no obvious changes in MxPIN1 and MxPIN10 expression occurred in response to IBA treatment in Mx-A cuttings (Xu et al. 2017), which is consistent with the higher GABA content and MxGADs expression in Mx-A cuttings. These results therefore imply that GABA might affect AR formation by inhibiting expression of MxPINs and subsequent polar auxin transport.

Though MxPIN12 is also homologous to long looped PINs, its expression did not respond to IBA, which was consistent with the previous result (Xu et al. 2017). As a possible reason, no AuxRR-core element (GGTCCAT) can be predicted in the promoter of MxPIN12 (Table S2). 
A

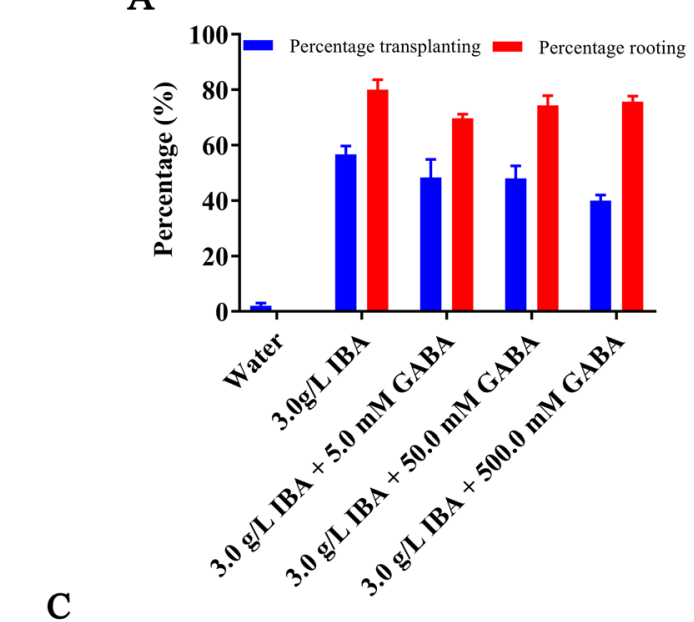

B

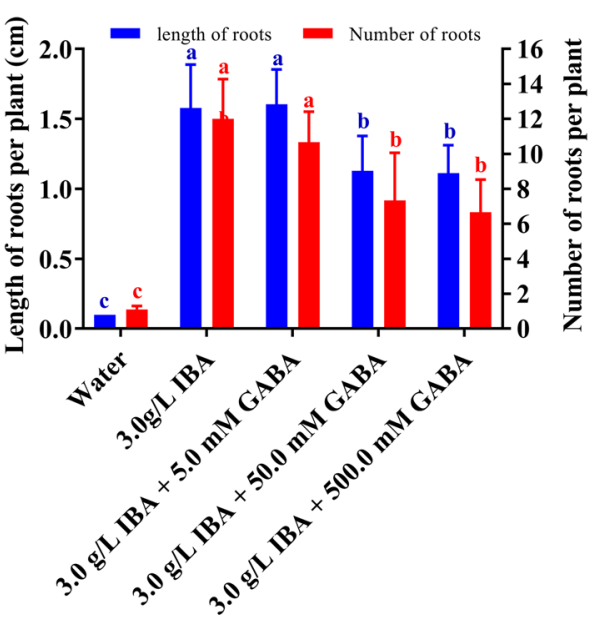

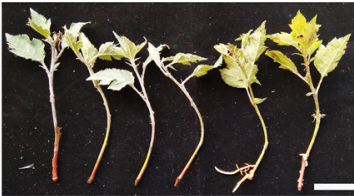

Water

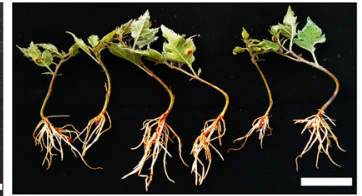

IBA

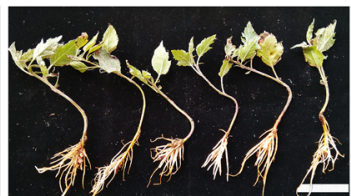

IBA +5.0 mM GABA

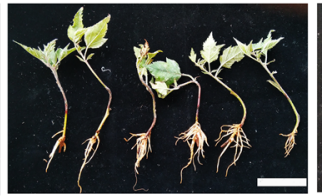

IBA+50.0 mM GABA

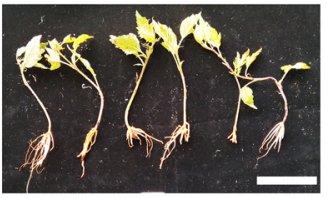

$I B A+500 \mathrm{mM} \mathrm{GABA}$
Fig. 4 Effects of exogenous $\gamma$-aminobutyric acid (GABA) on adventitious root formation in leafy cuttings of Malus xiaojinensis. a Differences in percentages of rooting and transplanting (more than three roots per plant) at the fifth week. b Differences in root number and root length per plant at the fifth week. c Differences in adventitious rooting at the fifth week
A

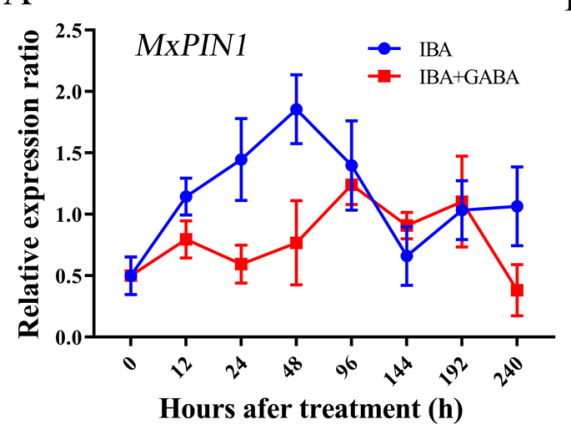

D

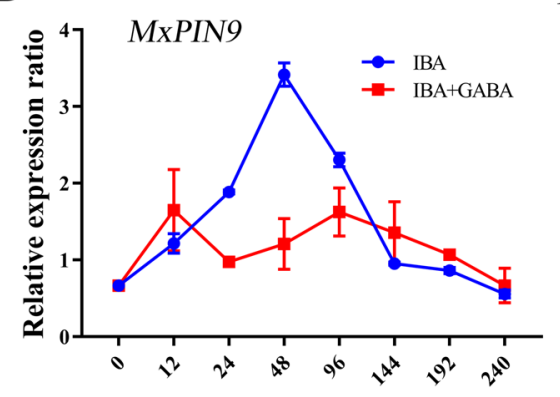

Hours after treatment (h)
B

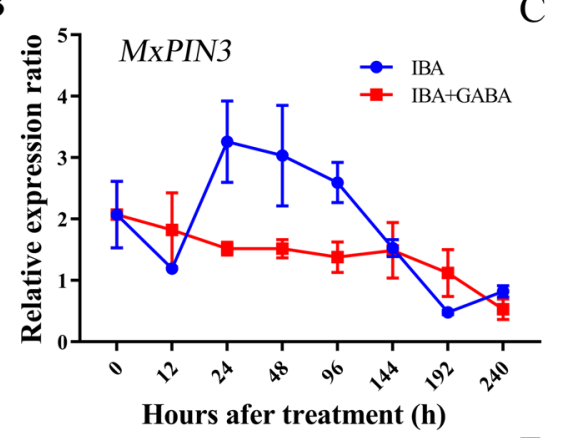

E

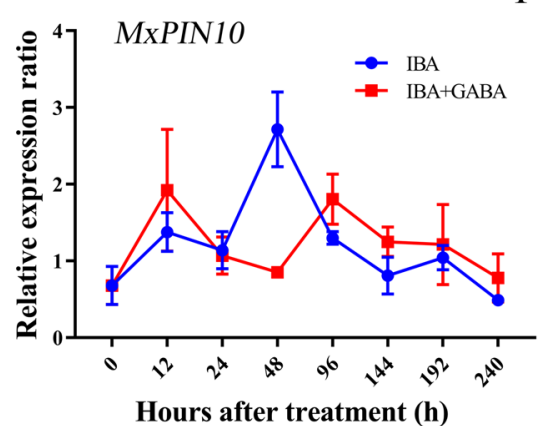

C

F
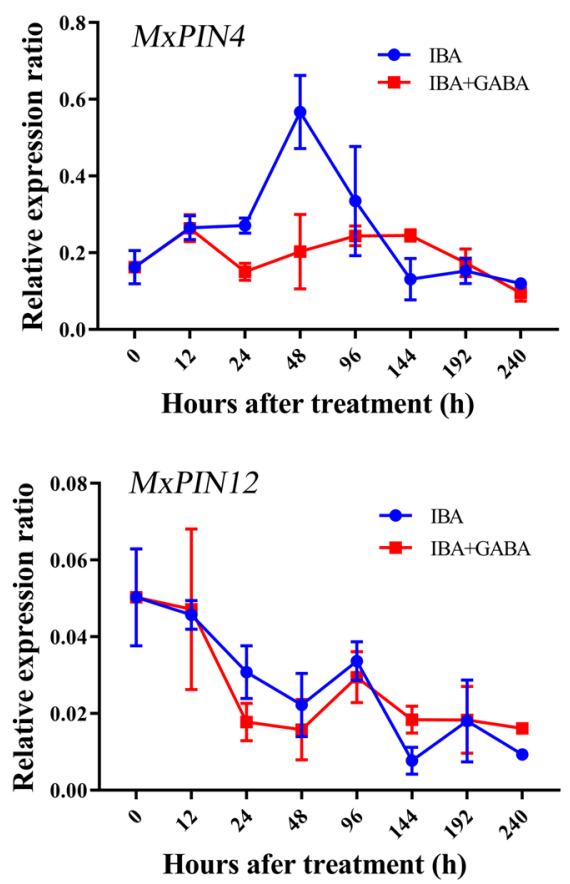

Fig. 5 Dynamic changes in expression of MxPINs during adventitious root formation after $\gamma$-aminobutyric acid (GABA) treatment in in vitro shoots of Malus xiaojinensis 
To date, there are two possible mechanisms explaining the effect of GABA on gene expression. In yeast, GABAinduced gene expression is mediated by several transcription factors including GATA factors (Cardillo et al. 2012). There are GATA-motifs on promoters of MxPIN4, MxPIN9, and MxPIN12, but not of MxPIN1, MxPIN3, and MxPIN10 (Table S2), which is inconsistent with the GATA motif mechanism. Alternatively, a GABA receptor, an aluminumactivated malate transporter (ALMT), was recently identified (Ramesh et al. 2015). In wheat, binding of GABA to the GABA-motif of ALMTs attenuates malate efflux ability of ALMTs (Ramesh et al. 2018). GABA and malate interact with ALMTs in a complex manner to regulate each other's transport, which results in altered root growth and tolerance to environmental stresses (Ramesh et al. 2015, 2017, 2018). Thus, the crosstalk of PINs, GABA signaling, and malate in AR formation needs future research (Xu et al. 2017; Podlesakova et al. 2019).

In both $M$. xiaojinensis and tobacco, GABA application led to delayed AR formation and reduced the AR number and root length in a dose-dependent manner. These results are perfectly consistent with previous research in Arabidopsis mutant and poplar transgenic lines, where GABA accumulation defected cell elongation and delayed AR primordium formation (Renault et al. 2011; Xie et al. 2020). These indicated that the inhibitory effect of GABA on AR formation is conserved between AR-recalcitrant and easy-toroot species, and also between woody perennial and annual herbaceous plants. However, the findings might not be sufficient to completely explain the adult-phase-specific rooting recalcitrance because of several adult-phase soft-wood cuttings are difficult-to-root or rooted very poorly regardless of auxin application (Diaz-Sala et al. 2010; Xiao et al. 2014; Aumond et al. 2017; Xu et al. 2017). GABA application did not mimic this serious AR recalcitrance in both $\mathrm{Mx}-\mathrm{J}$ and tobacco. Therefore, it was obvious that GABA might act as a co-factor, instead of a determinant, participating the adultphase-specific AR recalcitrance.

\section{Conclusion}

In AR-recalcitrant M. xiaojinensis, GABA accumulated in response to exogenous auxin treatment in Mx-A instead of Mx-J cuttings. GABA application inhibited the AR formation by delaying the rooting time and reducing the AR quantity in both rooting-recalcitrant woody perennial $M$. xiaojinensis and tobacco. GABA application also inhibited the auxin-induced upregulation of MxPINs. Together, these findings revealed functional details of GABA in AR formation, which sheds light on the understanding of the regulatory network among GABA, juvenility, and auxin signaling.
Acknowledgements We thank the supports of the Construction of Beijing Science and Technology Innovation and Service Capacity in Top Subjects (CEFF-PXM2019_014207_000032).

Author Contributions XL and XX performed the lab experiments. FS did the bioinformatic analysis. WL., CQ, TW, YW, and XX prepared the plant materials and participated lab experiments. $\mathrm{XZ}$ and $\mathrm{ZH}$ designed and supervised the experiments. XL wrote the draft and revised the manuscript.

Funding This work was supported by the National Natural Science Foundation of China (Grant Number: 31672107, 31872053, and 31801824).

\section{Compliance with ethical standarrds}

Disclosures All authors have read the manuscript and approved to submit.

Open Access This article is licensed under a Creative Commons Attribution 4.0 International License, which permits use, sharing, adaptation, distribution and reproduction in any medium or format, as long as you give appropriate credit to the original author(s) and the source, provide a link to the Creative Commons licence, and indicate if changes were made. The images or other third party material in this article are included in the article's Creative Commons licence, unless indicated otherwise in a credit line to the material. If material is not included in the article's Creative Commons licence and your intended use is not permitted by statutory regulation or exceeds the permitted use, you will need to obtain permission directly from the copyright holder. To view a copy of this licence, visit http://creativecommons.org/licenses/by/4.0/.

\section{References}

Akama K, Takaiwa F (2007) C-terminal extension of rice glutamate decarboxylase (OsGAD2) functions as an autoinhibitory domain and overexpression of truncated mutant results in the accumulation of extremely high levels of GABA in plant cells. J Exp Bot 58:2699-2707

Al-Taher F, Nemzer B (2019) Determination of gamma-aminobutyric acid (GABA) content in grains and cruciferous vegetable seeds. J Food Res 8:49-54

Anegawa A, Ohnishi M, Takagi D, Miyake C, Shichijo C, Ishizaki K, Fukaki H, Mimura T (2015) Altered levels of primary metabolites in response to exogenous indole-3-acetic acid in wild type and auxin signaling mutants of Arabidopsis thaliana: a capillary electrophoresis-mass spectrometry analysis. Plant Biotechnol J 32:65-79

Aumond ML Jr, de Araujo Jr AT, de Oliveira JCF, de Almeida MR, Matsuura HN, de Costa F, Fett-Neto AG (2017) Events associated with early age-related decline in adventitious rooting competence of Eucalyptus globulus Labill. Front Plant Sci 8:1734

Bach B, Meudec E, Lepoutre JP, Rossignol T, Blondin B, Dequin S, Camarasa C (2009) New insights into $\gamma$-aminobutyric acid catabolism: evidence for $\gamma$-hydroxybutyric acid and polyhydroxybutyrate synthesis in Saccharomyces cerevisiae. Appl Environ Microbiol 75:4231-4239

Behar TN, Schaffner AE, Scott CA, O’Connell C, Barker JL (1998) Differential response of cortical plate and ventricular zone cells to GABA as a migration stimulus. J Neurosci 18:6378-6387 
Benkova E, Michniewicz M, Sauer M, Teichmann T, Seifertova D, Jurgens G, Friml J (2003) Local, efflux-dependent auxin gradients as a common module for plant organ formation. Cell 115:591-602

Bouche N, Fromm H (2004) GABA in plants: just a metabolite? Trends Plant Sci 9:110-115

Bouche N, Lacombe B, Fromm H (2003) GABA signaling: a conserved and ubiquitous mechanism. Trends Cell Biol 13:607-610

Bown AW, Shelp BJ (1997) The metabolism and functions of [gamma]aminobutyric acid. Plant Physiol 115:1

Bown AW, Shelp BJ (2016) Plant GABA: not just a metabolite. Trends Plant Sci 21:811-813

Brikis CJ, Zarei A, Chiu GZ, Deyman KL, Liu J, Trobacher CP, Hoover GJ, Subedi S, DeEll JR, Bozzo GG, Shelp BJ (2018) Targeted quantitative profiling of metabolites and gene transcripts associated with 4-aminobutyrate (GABA) in apple fruit stored under multiple abiotic stresses. Hortic Res 5:61

Cardillo SB, Levi CE, Moretti MB, Garcia SC (2012) Interplay between the transcription factors acting on the GATA- and GABA-responsive elements of Saccharomyces cerevisiae UGA promoters. Microbiology 158:925-935

Che-Othman MH, Jacoby RP, Millar AH, Taylor NL (2020) Wheat mitochondrial respiration shifts from the tricarboxylic acid cycle to the GABA shunt under salt stress. New Phytol 225:1047-1048

da Costa CT, de Almeida MR, Ruedell CM, Schwambach J, Maraschin FS, Fett-Neto AG (2013) When stress and development go hand in hand: main hormonal controls of adventitious rooting in cuttings. Front Plant Sci 4:133

Da L, Liu Y, Yang J, Tian T, She J, Ma X, Xu W, Su Z (2019) AppleMDO: a multi-dimensional omics database for apple coexpression networks and chromatin states. Front Plant Sci 10:1333

Dawood T, Yang XP, Visser EJ, Te Beek TA, Kensche PR, Cristescu SM, Rieu I (2016) A co-opted hormonal cascade activates dormant adventitious root primordia upon flooding in Solanum dulcamara. Plant Physiol 170:2351-2364

Diaz-Sala C, Hutchison KW, Goldfarb B, Greenwood MS (2010) Maturation-related loss in rooting competence by loblolly pine stem cuttings: the role of auxin transport, metabolism and tissue sensitivity. Physiol Plant 97:481-490

Druege U, Hilo A, Pérez-Pérez JM, Klopotek Y, Acosta M, Shahinnia F, Zerche S, Franken P, Hajirezaei MR (2019) Molecular and physiological control of adventitious rooting in cuttings: phytohormone action meets resource allocation. Ann Bot 123:929-949

Fait A, Fromm H, Walter D, Galili G, Fernie AR (2008) Highway or byway: the metabolic role of the GABA shunt in plants. Trends Plant Sci 13:14-19

Ford YY, Ratcliffe RG, Robins RJ (1996) Phytohormone-induced GABA production in transformed root cultures of Datura stramonium: an in vivo ${ }^{15} \mathrm{~N}$ NMR study. J Exp Bot 47:811-818

Gan Z, Wang Y, Wu T, Xu X, Zhang X, Han Z (2018) MdPIN1b encodes a putative auxin efflux carrier and has different expression patterns in BC and M9 apple rootstocks. Plant Mol Biol 96:353-365

Ganguly A, Lee SH, Cho M, Lee OR, Yoo H, Cho H-T (2010) Differential auxin-transporting activities of PIN-FORMED proteins in Arabidopsis root hair cells. Plant Physiol 153:1046-1061

Han KL, Lee Y, Song JH, Hwang YS, Lee WS, Kim MW, Kim SH (2012) Enhanced production and secretion of rutin and GABA in immobilized cells of mulberry tree (Morus bombycis K.). Plant Cell Tissue Org 108:513-520

Jia XL, Chen YK, Xu XZ, Shen F, Zheng QB, Du Z, Wang Y, Wu T, Xu XF, Han ZH, Zhang XZ (2017) miR156 switches on vegetative phase change under the regulation of redox signals in apple seedlings. Sci Rep 7:14223-14237

Jin X, Liu T, Xu J, Gao Z, Hu X (2019) Exogenous GABA enhances muskmelon tolerance to salinity-alkalinity stress by regulating redox balance and chlorophyll biosynthesis. BMC Plant Biol 19:48

Kinnersley AM, Turano FJ (2000) Gamma aminobutyric acid (GABA) and plant responses to stress. Crit Rev Plant Sci 19:479-509

Krecek P, Skupa P, Libus J, Naramoto S, Tejos R, Friml J, Zazimalova E (2009) The PIN-FORMED (PIN) protein family of auxin transporters. Genome Biol 10:249

Li JB, Zhang J, Jia HX, Liu B, Sun P, Hu JJ, Wang LJ, Lu MZ (2017) The WUSCHEL-related homeobox 5a (PtoWOX5a) is involved in adventitious root development in poplar. Tree Physiol 38:139-153

Liu J, Sheng L, Xu Y, Li J, Yang Z, Huang H, Xu L (2014) WOX11 and 12 are involved in the first-step cell fate transition during de novo root organogenesis in Arabidopsis. Plant Cell 26:1081-1093

Liu S, Yang C, Wu L, Cai H, Li HG, Xu M (2020) The peu-miR160aPeARF 17.1/PeARF17.2 module participates in the adventitious root development of poplar. Plant Biotechnol J 18:457-469

Lum GB, Brikis CJ, Deyman KL, Subedi S, DeEll JR, Shelp BJ, Bozzo GG (2016) Pre-storage conditioning ameliorates the negative impact of 1-methylcyclopropene on physiological injury and modifies the response of antioxidants and g-aminobutyrate in 'Honeycrisp' apples exposed to controlled-atmosphere conditions. Postharvest Biol Technol 116:115-128

Luo H, Song J, Toivonen P, Gong Y, Forney C, Palmer LC, Fillmore D, Pang XQ, Zhang ZQ (2018) Proteomic changes in 'Ambrosia' apple fruit during cold storage and in response to delayed cooling treatment. Postharvest Biol Technol 137:66-76

Lü YG, Zhang H, Meng XY, Wang L, Guo X (2010) A validated HPLC method for the determination of GABA by pre-column derivatization with 2, 4-dinitrofluorodinitrobenzene and its application to plant GAD activity study. Anal Lett 43:2663-2671

Mekonnen DW, Flügge UI, Ludewig F (2016) Gamma-aminobutyric acid depletion affects stomata closure and drought tolerance of Arabidopsis thaliana. Plant Sci 245:25-34

\section{C- and N-metabolism}

Mustroph GAB, Kaiser KA, Larive CK, Bailey-Serres J (2014) Characterization of distinct root and shoot responses to low-oxygen stress in Arabidopsis with a focus on primary C- and N-metabolism. Plant Cell Environ 37:2366-2380

Nayyar H, Kaur R, Kaur S, Kaur S, Singh R (2014) $\gamma$-aminobutyric acid (GABA) imparts partial protection from heat stress injury to rice seedlings by improving leaf turgor and upregulating osmoprotectants and antioxidants. J Plant Growth Regul 33:408-419

Oh SJ, Kim HS, Lim ST (2019) Increase of gamma-aminobutyric acid contents in rice embryo with protein hydrolysates and pyridoxal5-phosphate using abiotic stress. J Cereal Sci 89:102803

Palanivelu R, Brass L, Edlund AF, Preuss D (2003) Pollen tube growth and guidance is regulated by $P O P 2$, an Arabidopsis gene that controls GABA levels. Cell 114:47-59

Podlesakova K, Ugena L, Spíchal L, Dolezal K, Diego DN (2019) Phytohormones and polyamines regulate plant stress responses by altering GABA pathway. New Biotechnol 48:53-65

Porfirio S, da Silva MDRG, Cabrita MJ, Azadi P, Peixe A (2016) Reviewing current knowledge on olive (Olea europaea L.) adventitious root formation. Sci Hortic 198:207-226

Ramesh SA, Kamran M, Sullivan W, Chirkova L, Okamoto M, Degryse F, McLaughlin M, Gilliham M, Tyerman SD (2018) Aluminumactivated malate transporters can facilitate GABA transport. Plant Cell 30:1147-1164

Ramesh SA, Tyerman SD, Gilliham M, Xu B (2017) $\gamma$-Aminobutyric acid (GABA) signaling in plants. Cell Mol Life Sci 74:1577-1603

Ramesh SA, Tyerman SD, Xu B, Bose J, Kaur S, Conn V, Domingos P, Ullah S, Wege S, Shabala S, Feijo JA, Ryan PR, Gilliham M (2015) GABA signalling modulates plant growth by directly 
regulating the activity of plant-specific anion transporters. Nat Commun 6:7879

Ramos-Ruiz R, Martinez F, Knauf-Beiter G (2019) The effects of GABA in plants. Cogent Food Agric 5:1670553

Ramos-Ruiz R, Poirot E, Flores-Mosquera M (2018) GABA, a nonprotein amino acid ubiquitous in food matrices. Cogent Food Agric 4:53-63

Rasmussen A, Hosseini SA, Hajirezaei MR, Druege U, Geelen D (2015) Adventitious rooting declines with the vegetative to reproductive switch and involves a changed auxin homeostasis. J Exp Bot 66:1437-1452

Renault H, Amrani AE, Palanivelu R, Updegraff EP, Yu A, Renou J, Preuss D, Bouchereau A, Deleu C (2011) GABA accumulation causes cell elongation defects and a decrease in expression of genes encoding secreted and cell wall-related proteins in Arabidopsis thaliana. Plant Cell Physiol 52:894-908

Salah A, Zhan M, Cao C, Han YL, Ling L, Liu ZH, Li P, Ye M, Jiang Y (2019) $\gamma$-Aminobutyric acid promotes chloroplast ultrastructure, antioxidant capacity, and growth of waterlogged maize seedlings. Sci Rep 9:484

Shelp BJ, Bozzo GG, Trobacher CP, Zarei A, Deyman KL, Brikis CJ (2012) Hypothesis/review: contribution of putrescine to 4-aminobutyrate (GABA) production in response to abiotic stress. Plant Sci 193-194:130-135

Sheteiwy MS, Shao H, Qi W, Hamoud YA, Shaghaleh H, Khan NU, Yang R, Tang B (2019) GABA-alleviated oxidative injury induced by salinity, osmotic stress and their combination by regulating cellular and molecular signals in rice. Int J Mol Sci 20:5709

Sukumar P, Maloney GS, Muday GK (2013) Localized induction of the ATP-binding cassette B19 auxin transporter enhances adventitious root formation in Arabidopsis. Plant Physiol 162:1392-1405

Vijayakumari K, Puthur JT (2016) $\gamma$-Aminobutyric acid (GABA) priming enhances the osmotic stress tolerance in Piper nigrum Linn. plants subjected to PEG-induced stress. Plant Growth Regul 78:57-67
Wang YC, Gu WR, Meng Y, Xie TL, Li J, Wei S (2017) $\gamma$-Aminobutyric acid imparts partial protection from salt stress injury to maize seedlings by improving photosynthesis and upregulating osmoprotectants and antioxidants. Sci Rep 7:43609

Xiao ZF, Ji N, Zhang XZ, Zhang YZ, Wang Y, Wu T, Xu XF, Han ZH (2014) The loss of juvenility elicits adventitious rooting recalcitrance in apple rootstocks. Plant Cell Tissue Org 119:51-63

Xie T, Ji J, Chen W, Yue J, Du C, Sun J, Shi S (2020) GABA negatively regulates adventitious root development. J Exp Bot 71:1459-1474

$\mathrm{Xu} \mathrm{L}$ (2017) De novo root regeneration from leaf explants: wounding, auxin, and cell fate transition. Curr Opin Plant Biol 41:39-45

Xu ML, Hu T, Zhao J, Park MY, Earley KW, Wu G, Poethig RS (2016) Developmental functions of miR156-regulated SQUAMOSA PROMOTER BINDING PROTEIN-LIKE (SPL) genes in Arabidopsis thaliana. PLoS Genet 12:e1006263

Xu XZ, Li X, Hu XW, Wu T, Wang Y, Xu XF, Zhang XZ, Han ZH (2017) High miR156 expression is required for auxin-induced adventitious root formation via MxSPL26 independent of PINs and ARFs in Malus xiaojinensis. Front Plant Sci 8:1059

Yue JY, Du CJ, Ji J, Xie TT, Chen W, Chang EM, Chen LZ, Jiang ZP, Shi SQ (2018) Inhibition of $\alpha$-ketoglutarate dehydrogenase activity affects adventitious root growth in poplar via changes in GABA shunt. Planta 248:963-979

Zarei A, Trobacher CP, Cooke AR, Meyers AJ, Hall JC, Shelp BJ (2015) Apple fruit copper amine oxidase isoforms: Peroxisomal MdAO1 prefers diamines as substrates, whereas extracellular MdAO2 exclusively utilizes monoamines. Plant Cell Physiol 56:137-147

Publisher's Note Springer Nature remains neutral with regard to jurisdictional claims in published maps and institutional affiliations. 